\title{
Reflexiones sobre montículos, técnicas de procesamiento de alimentos y construcción de nichos
}

\section{Roberto Bracco Boksar}

(iD) https://orcid.org/0000-0002-1686-7392

Ministerio de Educación y Cultura (MEC) - Facultad de Humanidades y Ciencias, Universidad de la República. Av. Uruguay 1695 (CP 11200), Montevideo, Uruguay. E-mail: braccoboksar@montevideo.com.uy

\section{Christopher Duarte}

\section{(iD) https://orcid.org/0000-0002-2801-7106}

Unidad de Ciencias de la Epigénesis (UNCIEP), Instituto de Ecología y Ciencias Ambientales, Facultad de Ciencias, Universidad de la República. Iguá 4225 (CP 11400), Montevideo, Uruguay. E-mail: christopherduarte1@gmail.com

\section{Ofelia Gutiérrez}

\section{(iD) https://orcid.org/0000-0002-1210-9658}

Unidad de Ciencias de la Epigénesis (UNCIEP), Instituto de Ecología y Ciencias Ambientales, Facultad de Ciencias, Universidad de la República. Iguá 4225 (CP 11400), Montevideo, Uruguay. E-mail: oguti@fcien.edu.uy

\section{Mario Clara}

\author{
(iD) https://orcid.org/0000-0002-5283-7270 \\ Centro Universitario de Rivera - Instituto de Ecología y Ciencias Ambientales, Facultad de Ciencias, Universidad de la \\ República. Iguá 4225 (CP 11400), Montevideo, Uruguay. E-mail: mclara@fcien.edu.uy

\section{Daniel Panario}

\section{(iD) https://orcid.org/0000-0001-7018-8289} \\ Unidad de Ciencias de la Epigénesis (UNCIEP), Instituto de Ecología y Ciencias Ambientales, Facultad de Ciencias, \\ Universidad de la República. Iguá 4225 (CP 11400), Montevideo, Uruguay. E-mail: panari@fcien.edu.uy
}

\begin{abstract}
Resumen
En este trabajo ensayamos una aproximación a los montículos de la región de India Muerta/Paso Barranca, sector sur de la cuenca de la Laguna Merín, Uruguay (5500 1000 años AP) a través de la relación entre las técnicas de procesamiento de alimentos y la Teoría de la Construcción de Nichos (TCN). Por una cuestión de escala temporal, de registro arqueológico y de enfoque epistemológico, no realizamos un planteo centrado en la evolución biológica; no obstante, exploramos cómo el concepto de poscosecha y la TCN nos ofrecen una aproximación alternativa y holística a los montículos. El primero nos induce a hacer foco en aspectos poco atendidos de las técnicas de subsistencia de los grupos prehistóricos: la relación entre los recursos explotados y las formas de procesarlos, que en nuestro caso podría ser una de las principales causas que produjo la elevación de los montículos. Por su parte, la TCN nos proporciona un marco teórico que faculta a abordar dicha relación y el singular proceso que desencadenó, desde una perspectiva diacrónica e integral donde se conjuga lo biológico, lo ecológico y lo cultural.
\end{abstract}




\title{
Reflections about mounds, food processing techniques and niche construction
}

\begin{abstract}
In this paper, we test an approach to the India Muerta/Paso Barranca region mounds, located in the southern sector of laguna Merín basin, Uruguay (5500 - 1000 years BP), through the relationship between food processing techniques and Niche Construction Theory (NCT). Due to issues related to the temporal scale, the archaeological record, and an epistemological approach, we do not carry out a proposal focused on biological evolution. However, we explore how the postharvest concept and the NCT offer us an alternative and holistic approach to the mounds. The first concept leads us to focus on issues dealing with prehistoric group subsistence techniques that have not been properly addressed: the relationship between the exploited resources and the ways of processing them. This relationship, in our case, could be one of the principal causes that led to the elevation of the mounds. On the other hand, the NCT provides a theoretical framework that allows us to address this relationship and the singular process generated from that, taking into account a diachronic and integral perspective where biological, ecological, and cultural aspects are combined.
\end{abstract}

KEYWORDS: Indian mounds; Postharvest; Ecological heritage; Landscape construction.

\section{Introducción}

Los "cerritos de indios" son elevaciones artificiales del terreno hechas por las poblaciones originarias. También se los ha denominado aterros, tesos, terremotos, estructuras monticulares o simplemente montículos (Arechavaleta, 1892; Bracco, Cabrera y López Mazz, 2000; Cabrera, 2013; Copé, 1991; Ferrés, 1927; Gianotti, 2000; Iriarte et al., 2004; López Mazz, Rostain y McKey, 2016; Milheira, Attorre y Borges, 2019; Schmitz, 1981, 1976). En Uruguay y sur de Brasil, se distribuyen en las zonas inundables del bioma Pampa (Milheira y Gianotti, 2018) desde el extremo austral de la cuenca de la Laguna de los Patos hasta la cuenca de la Laguna de Castillos, incluyendo la cuenca media y superior del río Negro (Figura 1). En esta región el clima actual es subtropical húmedo con verano cálido, con una temperatura media anual de $17^{\circ} \mathrm{C}$ y una precipitación anual media de 1.300 mm (Serrentino, 2013). Los montículos exhiben plantas circulares o alargadas con un diámetro promedio de 35 metros y alturas que van desde algunos decímetros a siete metros (Figura 2). Se presentan aislados o más frecuentemente agrupados en dos posiciones del paisaje: bañados y colinas (Figura 3). En la primera, se ubican próximos a cursos anastomosados, predominantemente en las llanuras medias (actualmente de inundación esporádica). En la segunda, se sitúan preferentemente en el extremo de lomadas que se proyectan hacia el bañado. Al pie de éstas se encuentra frecuentemente un sector ensanchado del curso de un arroyo, lo que lleva a que se los denomine localmente "lagunas". Los tres sitios con mayor número de montículos de la región de India Muerta/Paso Barranca que reúnen aproximadamente 150 montículos (Los Ajos, MD y Campo Alto) se hallan al borde de la "Laguna" de Los Ajos y de la "Laguna" de Campo Alto (Bracco, Inda y del Puerto, 2015). A los montículos se les han atribuido diversas y/o múltiples funciones: plataformas para ocupar áreas inundables, marcadores territoriales, sitios de enterramiento, rituales, monumentos, demarcadores de espacios públicos ("plazas"), lugares de cultivo, lugares de descarte (Baeza y Panario, 1999; Bracco et al., 2000; Gianotti, 2000; Iriarte, 2006; Iriarte, Holst, López Mazz y Cabrera, 2000; López Mazz, 2001; Loureiro, 2008; Milheira y Gianotti, 2018; Schmitz, 1981; Villagran y Gianotti, 2013). Sus cronologías absolutas 


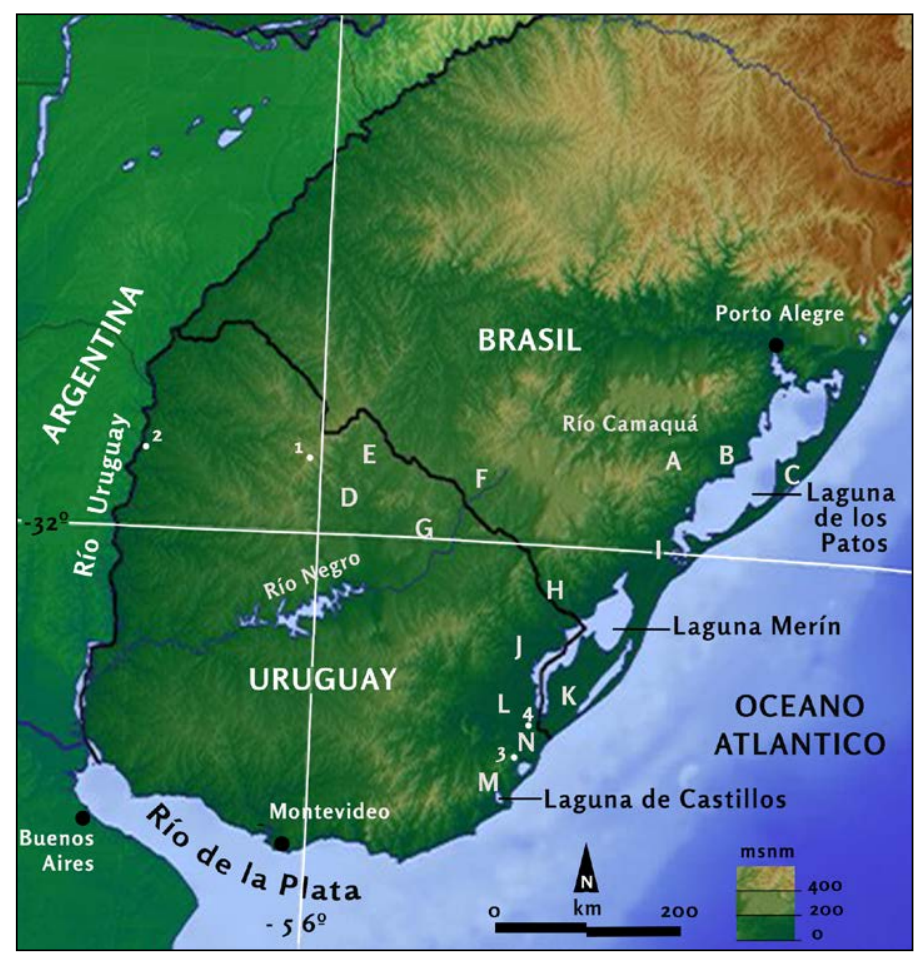

Figura 1. Cuenca de la laguna Merín. Concentración montículos: A) Sierra do Sudeste; B) Camaquã; C) Parte Central Planicie Costera Laguna de los Patos; D) Valle del Yaguarí; E) Rivera; F) Bagé-Dom Pedrito; G) Cerro Largo; H) Rio Jaguarão; I) Sur Laguna de los Patos; J) Treinta y Tres; K) Santa Vitoria do Palmar; L) India Muerta/Paso Barranca; M) Laquna de Castillos; y N) Bañado de San Miguel. Sitios arqueológicos: 1) Yacaré-Cururú; 2) Y 58; 3) Rincón de los Indios; y 4) CH2D01 (modificado de Bracco et al., 2020).

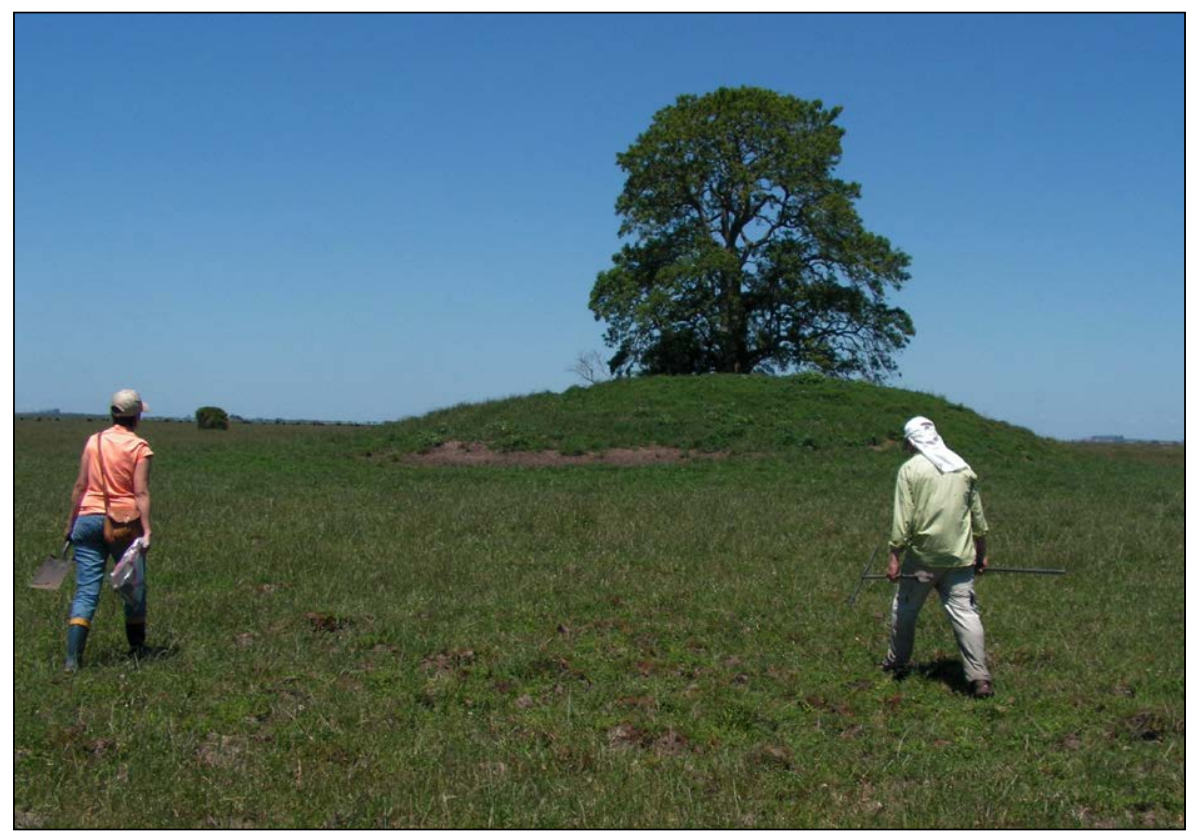

Figura 2. Montículo del sitio Pelotas sector norte de la región de India Muerta/Paso Barranca. 


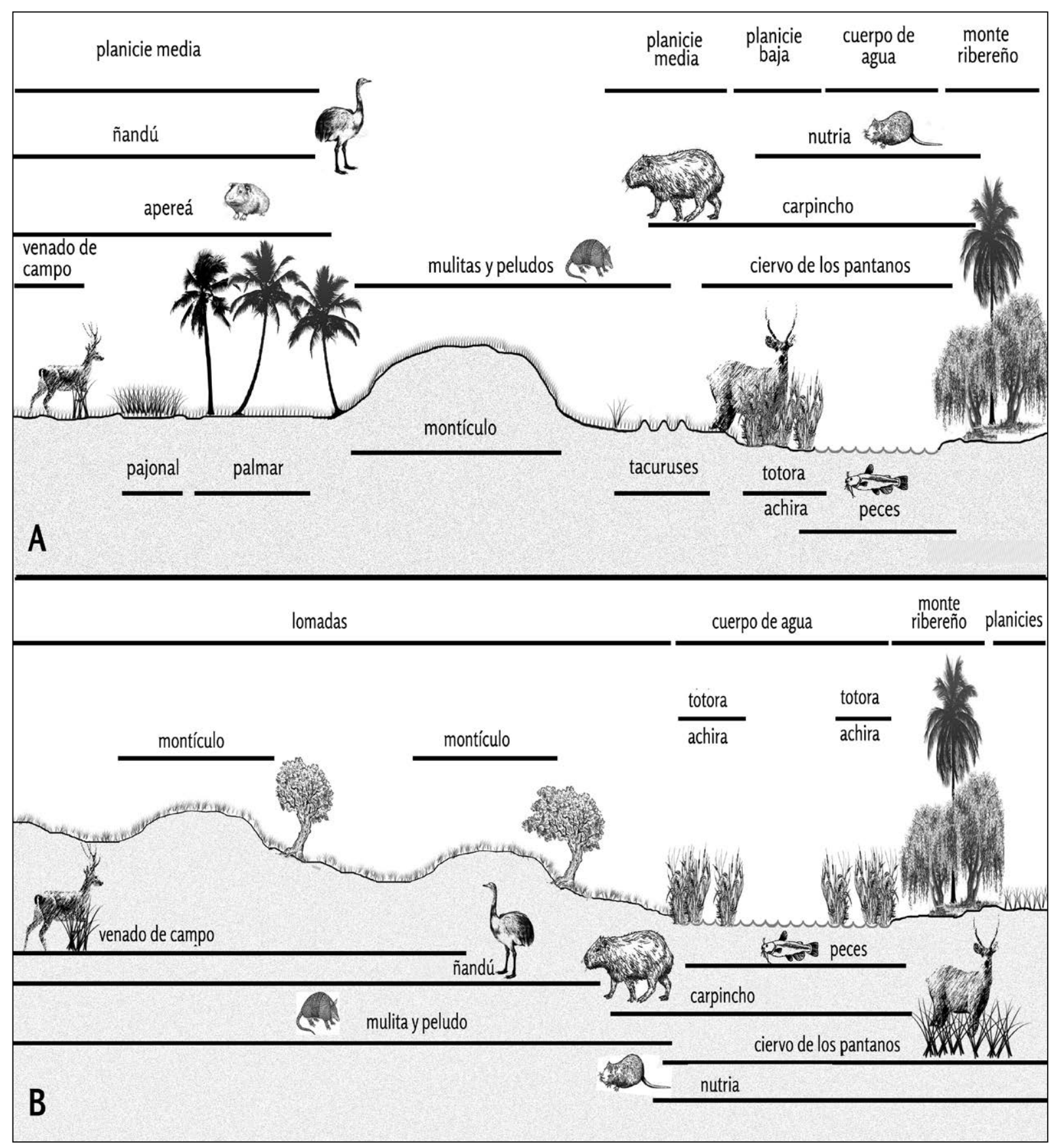

Figura 3. Representación esquemática de la posición de los montículos, la distribución de los recursos con mayor presencia en el registro arqueofaunístico (Moreno, 2014) y los geófitos de mayor oferta (totora y achira) en (A) bañados y (B) lomadas.

cubren el período 5500-200 años AP' (Bracco et al., 2015, tabla 1; Milheira et al., 2019). El hallazgo de material europeo en su interior corrobora que alcanzaron el período histórico (Cabrera y Marozzi, 2001; Pintos Blanco y Capdepont, 2001). Los fechados más antiguos proceden de India Muerta/Paso Barranca, región en la que se habrían originado (Bracco et al., 2015). También es en esta amplia zona de bañados y lomadas

1 A este lapso corresponden las cronologías de la cuenca de la Laguna Merín y la Laguna de los Patos, e incluye la región de India Muerta/Paso Barranca donde las cronologías más recientes alcanzan los 1000 años AP. 
donde se hallan los montículos más altos, donde se encuentran los agrupamientos más numerosos y donde se observa la mayor densidad para el extremo sur de su distribución (Tabla 1). Casi un tercio se concentra en las llanuras medias encharcadas, y más de la mitad en esa unidad y las llanuras medias continentales.

El objetivo del presente trabajo es ensayar una aproximación a los montículos de la región de India Muerta/Paso Barranca, sector sur de la cuenca de la Laguna Merín (CLM), Uruguay, relacionando las técnicas de procesamiento de alimentos y la Teoría de la Construcción de Nichos (TCN). No se realiza un planteo centrado en la evolución biológica, por una cuestión de escala temporal, de registro arqueológico y de enfoque epistemológico. No obstante, exploramos cómo la relación entre el concepto de poscosecha y la TCN nos proporciona una aproximación alternativa y holística a los montículos. El primero nos induce a hacer foco en aspectos de las técnicas de subsistencia de los grupos prehistóricos: la relación entre los recursos explotados y las formas de procesarlos que, en nuestro caso, podría ser una de las principales causas que produjo la elevación de los montículos. Por su parte, la TCN nos provee de un marco teórico para abordar el proceso de elevación de los montículos desde una perspectiva diacrónica e integral que conjuga lo biológico, lo ecológico y lo cultural.

Para cumplir este objetivo abordamos primero los modelos de subsistencia inferidos para los constructores de montículos del Uruguay. Luego tratamos el concepto de poscosecha y el vínculo que hemos propuesto entre montículos y hornos de tierra, resaltando su rol en la explotación de vegetales disponibles por largos períodos anuales, con alta previsibilidad y altas tasas de retorno. Se indican las posibles implicaciones socio-económicas de la adquisición o intensificación del uso de esta técnica. Finalmente, bajo el marco de la TCN, abordamos cómo una forma de procesar alimentos habría generado la transformación del paisaje.

\begin{tabular}{|l|c|c|c|c|}
\hline Unidad de paisaje & $\begin{array}{c}\text { Población de } \\
\text { montículos }\end{array}$ & Superficie km² & $\begin{array}{c}\text { Densidad de } \\
\text { montículos por km² }\end{array}$ & \% población \\
\hline Llanuras medias encharcadas & 201 & 243,91 & 0,82 & 31,60 \\
Llanuras medias continentales & 171 & 284,45 & 0,60 & 26,90 \\
Bañados & 100 & 301,88 & 0,33 & 15,72 \\
Lomadas & 116 & 30,17 & 3,85 & 18,24 \\
Otras & 48 & 532,59 & 0,09 & 7,55 \\
\hline Totales & 636 & 1393,16 & 0,46 & 100,0 \\
\hline
\end{tabular}

Tabla 1. Población y densidad de montículos en la región de India Muerta/Paso Barranca discriminadas por unidades de paisaje (Información tomada de Bracco et al., 2015 y J. Mazzarino, comunicación personal, 2019).

\section{Modelos de subsistencia de los constructores de montículos: dieta y vegetales}

En los primeros modelos de subsistencia propuestos (Bracco et al., 2000) para los grupos vinculados a los montículos del Uruguay, se prestó mayor atención a los recursos faunísticos que a los vegetales (ver López Mazz y Bracco, 1992; Pintos Blanco, $2000)^{2}$. Esta visión comenzó a modificarse parcialmente a medida que los registros arqueobotánicos ofrecieron pruebas de domesticación, particularmente de Zea mays,

2 Esto también sucedió para los montículos de Río Grande do Sul con mayor presencia en algunos casos de recursos litorales (ver Milheira et al., 2019; Schmitz, 1976; Schmitz, Girelli y Rosa, 1997; Schorr, 1975; entre otros). 
Cucurbitaceae sp. y Phaseolus sp. (del Puerto, 2015; del Puerto e Inda, 2005; Iriarte et al., 2004; Olivero y Campos, 2001). Pero los recursos vegetales silvestres se mantuvieron en un segundo plano (cf. del Puerto, 2015; Gianotti, del Puerto, Inda y Capdepont, 2013). Asimismo, el rol atribuido a los cultígenos quedó fuertemente condicionado al concepto de "sociedades complejas". Es así que, conjugando el cultivo o el manejo de vegetales con la interpretación de los montículos como expresiones arquitectónicas, se propone la existencia de asentamientos semipermanentes o permanentes y hasta de aldeas planificadas, evidencias de sociedades cazadoras-recolectoras complejas o de "sociedades formativas" (Bracco et al., 2000; Gianotti y Bonomo, 2013; Iriarte, 2006, 2007; Iriarte et al., 2004; López Mazz, 2001; López Mazz y Bracco, 1992; Milheira et al., 2019). Sin embargo, estas interpretaciones no son consistentes con otras dimensiones del registro y particularmente con indicadores de dieta (Bracco, 2006). Así, los primeros datos $\delta^{13} \mathrm{C}_{\text {colágeno }}$ humano señalaron una dieta continental basada en vía fotosintética $\mathrm{C}_{3}$ y no evidenciaron consumo de recursos marinos ni tampoco de plantas $\mathrm{C}_{4}$, por lo menos en cantidades significativas (Bracco et al., 2000). Estudios más recientes, que conjugaron otras variables isotópicas junto al registro arqueofaunístico y paleobotánico, han permitido una mayor aproximación al cuadro dietario (del Puerto, 2015; Mut, 2015). Los valores de $\delta^{15} \mathrm{~N}$ y $\delta^{13} \mathrm{C}_{\text {colágeno }}$ (Tabla 2$)^{3}$ confirman un consumo continental de proteínas. El registro arqueofaunístico revela que éstas provendrían principalmente del consumo de mamíferos terrestres, aunque también de peces. La importancia en la dieta de los cérvidos (Blastocerus dichotomus) y venados (Ozotoceros bezoarticus uruguayensis, Mazama gouazoubira) fue señalada por Pintos Blanco (2000). Moreno (2014) señala que el apereá (Cavia aperea) y la nutria (Myocastor coypus) también habrían jugado un papel importante. Por otra parte, para algunos individuos los valores $\Delta^{13} \mathrm{C}_{\text {colageno-apatito }}$ se encuentran dentro del extremo inferior del rango que corresponde a grupos que consumen alimentos de patrón fotosintético $C_{4}$ (Mut, 2015), lo que estaría señalando un consumo moderado de maíz. Ello es consistente con el registro microbotánico (del Puerto, 2015). La incidencia del maíz en la dieta pudo haberse incrementado con el tiempo (Figura 4). Igualmente no se puede descartar que la señal $\mathrm{C}_{4}$ se encuentre enmascarada por el consumo de plantas con valores $\delta^{13} \mathrm{C}$ muy empobrecidos, como es el caso de Typha: $-27 \%{ }^{4}$ (Inglett y Reddy, 2006). Su ingesta también explicaría los valores tan empobrecidos de $\delta^{13} \mathrm{C}_{\text {colágeno }}$. Por su parte Mut (2015) plantea que los valores $\delta^{13} \mathrm{C}_{\text {colágeno }}$ podrían deberse a la incidencia de los peces en la dieta. Ambas posibilidades no son excluyentes. En cuanto a la composición de la dieta, comparando datos $\delta^{15} \mathrm{~N}$ de humanos y fauna, Mut (2015) indica que el $78 \%$ de la proteína consumida habría sido de origen animal. Sin embargo, la autora señala la posibilidad de que el consumo de peces podría estar sobreestimando ese valor hasta un $20 \%$, pasando del 78 al $58 \%$. Hedges y Reynard (2007) han advertido sobre los errores que pueden cometerse al inferir el nivel trófico linealmente de los valores de $\Delta^{15} \mathrm{~N}$ (ver O'Connell, Kneale, Tasevska y Kuhnle, 2012). Por otra parte los valores de $\delta^{13} \mathrm{C}_{\text {colageno }}$ para humanos y fauna arqueológica local indicarían que los vegetales $\mathrm{C}_{3}$ fueron importantes en la ingesta (Mut, 2015). En forma consistente la distancia Sr/Ca entre restos óseos de animales y humanos indica una dieta rica en plantas (Bracco et al., 2000, figuras 3 y 4). Los datos expuestos permiten sustentar que estaríamos frente a una economía donde la caza, la pesca y la colecta se complementó con la horticultura a pequeña escala (Bracco, 2006; del Puerto, 2015; Mut, 2015), y donde el componente vegetal $\mathrm{C}_{3}$ conformó una proporción importante de la dieta. Sin desconocer que hay algunos indicadores que estarían mostrando cambios, al considerar los tiempos involucrados (5000 años) la opción económica se acerca más a una estrategia a largo plazo que a un tránsito hacia una economía productiva (Greaves y Kramer, 2014).

3 Las muestras 30 y 31 presentan $\mathrm{C} / \mathrm{N}$ fuera de los márgenes para colágeno no alterado (Ambrose, 1990; DeNiro, 1985), y la muestra 32 procedente del litoral de la laguna de Castillos, presenta valores ${ }^{13} \mathrm{C}_{\text {colageno }}$ y ${ }^{15} \mathrm{~N}$ anómalos para la serie (datos que son eliminados).

4 Este valor es actual, corregido por efecto Suess (+1,5\%) es -28,5\% (Francey et al., 1999). 


\begin{tabular}{|c|c|c|c|c|c|c|c|c|c|}
\hline$\#$ & Sitio & Sexo & Fechados (AP) & ${ }_{(\%)}^{\delta^{13} C_{\text {fol }}}$ & $\begin{array}{l}\delta^{13} C_{\text {Apa }} \\
(\%)^{-1}\end{array}$ & $\begin{array}{l}\delta^{15} \mathrm{~N} \\
(\%)\end{array}$ & $\begin{array}{c}\delta 13 C_{(\%)} \text { Clipa } \\
(\%)\end{array}$ & $\% \mathrm{C}: \% \mathrm{~N}$ & Fuente \\
\hline 1 & $\mathrm{CH} 2 \mathrm{D} 01-\mathrm{A}$ & Masc. & $290 \pm 75^{\star}$ & $-19,3$ & - & - & - & - & (1) \\
\hline 2 & $\mathrm{CH} 2 \mathrm{D} 01-\mathrm{A}$ & Niño & $400 \pm 50$ & $-19,5$ & - & - & - & - & (1) \\
\hline 3 & CH2D01-A & Masc. & $400 \pm 50$ & $-20,5$ & - & - & - & - & (1) \\
\hline 4 & $\mathrm{CH} 2 \mathrm{D} 01-\mathrm{B}$ & Masc. & $1090 \pm 70$ & $-18,5$ & - & - & - & - & $(1)$ \\
\hline 5 & CH2D01-B & Niño & $1090 \pm 70$ & $-19,8$ & - & - & - & - & (1) \\
\hline 6 & CH2D01-B & Fem. & $1090 \pm 70$ & $-20,4$ & - & - & - & - & (1) \\
\hline 7 & CH2D01-B & - & $1090 \pm 70$ & $-20,6$ & - & - & - & - & (1) \\
\hline 8 & $\mathrm{CH} 2 \mathrm{D} 01-\mathrm{B}$ & - & $2530 \pm 60$ & $-19,0$ & - & - & - & - & (1) \\
\hline 9 & $\mathrm{CH} 2 \mathrm{D} 03$ & - & - & $-21,2$ & - & - & - & - & (1) \\
\hline 10 & Santiagueño & - & - & $-20,7$ & - & - & - & - & (1) \\
\hline 11 & India Muerta & - & $1590 \pm 90$ * & $-21,3$ & $-11,6$ & - & 9,7 & - & (1) \\
\hline 12 & India Muerta & - & - & $-21,4$ & - & - & - & - & (1) \\
\hline 13 & India Muerta & - & - & $-20,9$ & - & - & - & - & (1) \\
\hline 14 & CH2D01-B & Fem. & $1350 \pm 160$ & $-20,6$ & $-8,6$ & 9,2 & 12,0 & 2,9 & (2) \\
\hline 15 & CG14E01 & Masc. & - & $-20,5$ & $-9,8$ & 9,8 & 10,7 & 2,7 & $(2)$ \\
\hline 16 & CG14E01 & Fem. & - & $-20,8$ & $-9,3$ & 9,7 & 11,5 & 2,8 & $(2)$ \\
\hline 17 & CH2D01-A & Masc. & - & $-21,6$ & $-12,2$ & 9,2 & 9,4 & 3,1 & $(2)$ \\
\hline 18 & $\mathrm{CH} 2 \mathrm{D} 01-\mathrm{A}$ & Masc. & - & $-20,6$ & $-9,0$ & 9,4 & 11,6 & 3,0 & $(2)$ \\
\hline 19 & $\mathrm{CH} 2 \mathrm{D} 01-\mathrm{A}$ & Masc. & - & $-20,9$ & $-11,8$ & 9,8 & 9,1 & 2,8 & $(2)$ \\
\hline 20 & $\mathrm{CH} 2 \mathrm{D} 01-\mathrm{A}$ & Fem. & $1610 \pm 46$ & $-21,6$ & $-12,3$ & 9,6 & 9,2 & 2,6 & (2) \\
\hline 21 & CH2D01-A & Masc. & $1000 \pm 85$ & $-21,5$ & $-11,8$ & 9,6 & 9,7 & 2,7 & (2) \\
\hline 22 & $\mathrm{CH} 2 \mathrm{D} 01-\mathrm{A}$ & - & $778 \pm 45$ * & $-21,6$ & - & 9,54 & - & 2,8 & $(3)$ \\
\hline 23 & $\mathrm{CH} 2 \mathrm{D} 01-\mathrm{A}$ & - & $700 \pm 45$ * & $-21,0$ & - & 10,1 & - & 2,9 & $(3)$ \\
\hline 24 & CG14E01 & Masc. & $830 \pm 70 *$ & $-21,2$ & - & 10,1 & - & 2,8 & (3) \\
\hline 25 & CG14E01 & Fem. & $850 \pm 70$ * & $-21,3$ & - & 9,99 & - & 2,7 & $(3)$ \\
\hline 26 & PSLIID1 & - & $1390 \pm 90$ * & $-21,2$ & - & 11,4 & - & 2,9 & $(3)$ \\
\hline 27 & PSLIID1 & - & $1360 \pm 100^{*}$ & $-21,2$ & - & 11,4 & - & 2,9 & $(3)$ \\
\hline 28 & PSLII-III & - & $1470 \pm 90$ * & $-21,0$ & - & 10,3 & - & 2,8 & (3) \\
\hline 29 & C.MIIIB & Fem. & - & $-20,7$ & - & 9,89 & - & 3,0 & (3) \\
\hline 30 & C.MIIIB & - & - & $-20,3$ & - & 12,4 & - & 5,0 & (3) \\
\hline 31 & C.MIIIB & Fem. & $980 \pm 100$ * & $-20,4$ & - & 10,1 & - & 4,4 & (3) \\
\hline 32 & C.MIIIB & - & - & $-14,2$ & - & 18,6 & - & 2,9 & (3) \\
\hline \multicolumn{2}{|r|}{$x(1-32)=$} & - & - & $-20,478$ & - & 10,533 & - & - & - \\
\hline \multicolumn{2}{|r|}{$s(1-32)=$} & - & - & 1,375 & - & 2,118 & - & - & - \\
\hline \multicolumn{2}{|r|}{$x(1-29)=$} & - & - & $-20,703$ & - & 9,939 & - & - & - \\
\hline \multicolumn{2}{|r|}{$s(1-29)=$} & - & - & 0,795 & - & 0,649 & - & - & - \\
\hline
\end{tabular}

Tabla 2. Datos isotópicos del este del Uruguay. Referencias: (*) fechados directos, el resto son la edad del nivel (Información tomada de Bracco et al., 2000 (1); del Puerto, 2015 (2); Mut, 2015 (3)). Se incluyen los valores medios y desviación estándar para toda la serie y para la serie eliminando datos anómalos. Resaltados en negrita y sombreado los datos $\mathrm{C} / \mathrm{N}$ fuera de los márgenes para colágeno no alterado. 


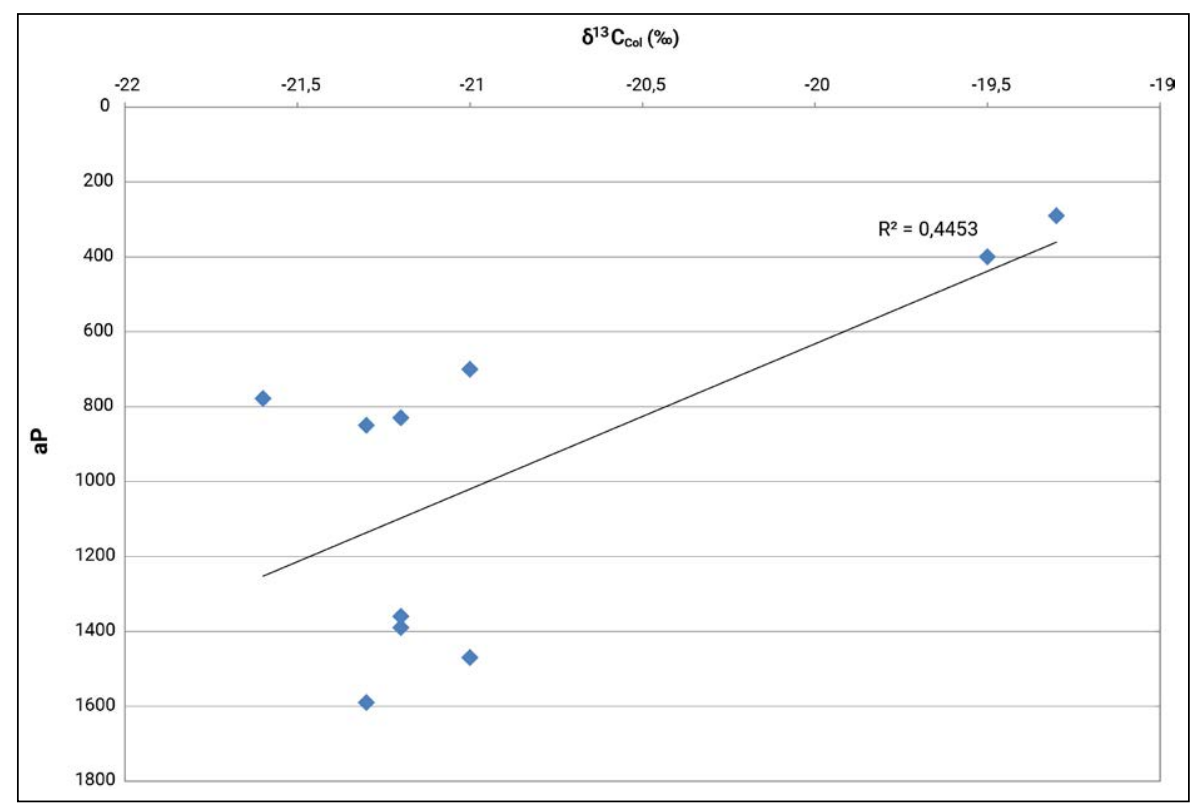

Figura 4. Valores $\delta^{13} \mathrm{C}$ humano/edad de las muestras que cuentan con dataciones directas (ver Tabla 2).

\section{Procesamiento de alimentos y poscosecha}

Los sistemas de subsistencia incluyen tanto las técnicas de aprovisionamiento como de manejo y preparación de alimentos. Estas últimas no sólo han ampliado la dieta de los homínidos sino que, a largo plazo, han incidido en la evolución biológica y socio-cultural de nuestra especie (Wrangham y Conklin-Brittain, 2003). No obstante, la gran y sorprendente diversidad que presentan las formas de preparar los alimentos, son concomitantes y articuladoras de contingencias ecológicas, socioeconómicas y culturales (Figura 5).

El procesamiento involucra dos grandes clases de prácticas: las que modifican físicamente los alimentos y las que provocan trasformaciones químicas en ellos. Dentro de las primeras tenemos el lavado, pelado, cortado, rallado y la molienda. Dentro de las segundas, la fermentación, desecación, el ahumado y la cocción. Lo más frecuente es que se empleen en forma combinada. Los cambios que ocasionan hacen que los alimentos sean más apetecibles, fáciles de ingerir, digerir, menos riesgosos y muy frecuentemente que algunos de sus nutrientes aumenten su biodisponibilidad (Wandsnider, 1997) ${ }^{5}$. El concepto de poscosecha nos permite analizar la lógica de esos procesos, remitiendo a las relaciones que existen entre las propiedades de los recursos y las tecnologías utilizadas para procesarlos (Wollstonecroft, 2011), particularmente cuando son vegetales por conllevar procesamientos más complejos. Por ejemplo, los almidones almacenados en grandes cantidades en los órganos sub-aéreos de los geófitos aumentan marcadamente su biodisponibilidad al ser cocidos por períodos prolongados, a temperaturas moderadas y en atmósfera húmeda. La presencia de sistemas de cocción que proporcionen esas condiciones de procesamiento nos estaría indicando el uso de este tipo de recursos vegetales. Y, aún más, un incremento del

5 Muchos nutrientes procedentes de especies silvestres o domesticadas no pueden ser absorbidos en su totalidad cuando se comen sin cocer (e.g. trigo, papa), para otros alimentos el procesamiento redunda en una disminución de sus riesgos sanitarios y de toxicidad a niveles aceptables (cerdo y mandioca amarga) y hasta para otros alargan los periodos de conservación (piñones) (Wandsnider, 1997). 


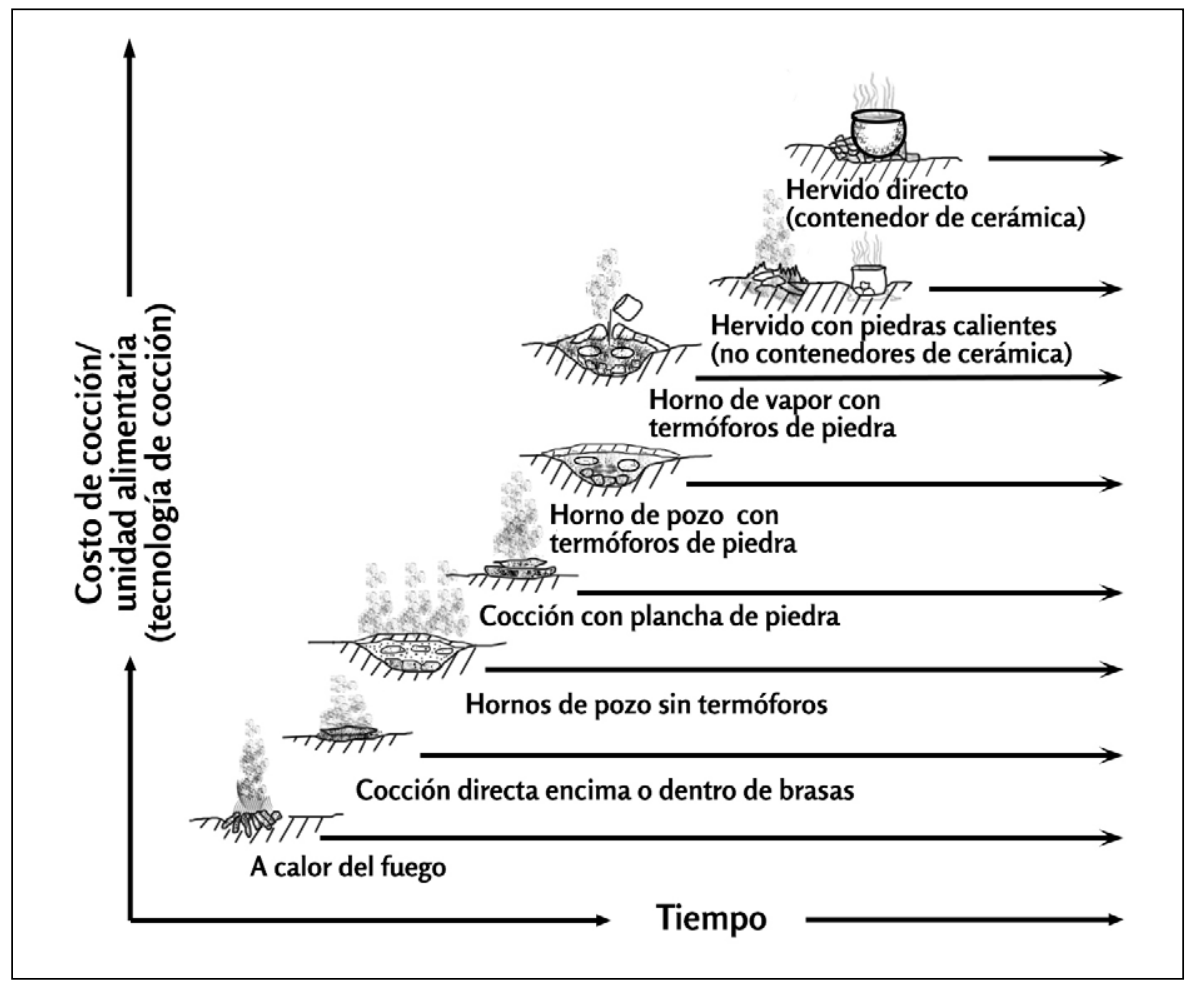

Figura 5. Modelo de la intensificación del uso del espacio y patrones temporales esperados de sistemas de cocción (basado en Thoms, 2008, figura 7).

uso de esos sistemas de cocción es un indicador de un proceso de intensificación basado en esos recursos. Como corolario, a través del concepto de poscosecha podemos - a partir de evidencias del empleo de determinadas prácticas de procesamiento-, acercarnos a cuáles recursos habrían sido explotados y en qué contexto socioeconómico habría sucedido (Thoms, 2008).

\section{Montículos y hornos de tierra}

La hipótesis que sostiene que los montículos de la región de India Muerta/Paso Barranca son la consecuencia de la acumulación de residuos producidos por el uso de hornos de tierra (HT) se basa principalmente en dos líneas de evidencias (Bracco et al., 2019a, 2019b; Bracco, Panario, Gutiérrez, Duarte y Bazzino, 2019c). Edades radiocarbónicas (Bracco et al., 2015, tabla 1) y luminiscentes (Bracco et al., 2019b; Duarte et al., 2017) revelan que los montículos crecieron en forma paulatina por períodos prolongados, en algunos casos durante milenios (Bracco, 2006; Bracco, del Puerto e Inda, 2008; Bracco y Ures, 1999; Duarte et al., 2017), no como producto de un proyecto planificado, sino más bien como consecuencia no esperada de un comportamiento (sensu Giddens, 1997, p. 33), en este caso recurrente. Por otra parte, la abundancia de tierra quemada y restos vegetales carbonizados, así como las características geoquímicas de matriz y particularmente sus registros luminiscentes, indican que el fuego tuvo una fuerte presencia durante la elevación (Bracco et al., 2019a, 2020). Particularmente la consistencia entre edades luminiscentes OSL y TL de fracciones arena y limo, procedentes de mismas profundidades, ha llevado a concluir que toda (o casi toda) la matriz fue calentada a temperaturas mínimas de $350^{\circ} \mathrm{C}$ (Bracco et al., 2019c). Esta hipótesis se reafirma con la inexistencia en los montículos de evidencias que manifiesten otras formas de construcción (cf. Gianotti et al., 2009; López Mazz, 
2000) ${ }^{6}$. Pastor Quiles, Knoll y Jover Maestre (2019) sistematizan las evidencias de estructuras analizables para las construcciones en las cuales se emplea tierra como material y donde el crecimiento se da por el agregado intencional de bloques, terrones, conos, bolas, etc. En tanto, en la elevación que proponemos se habrían desintegrado las estructuras originales, a excepción de los restos de nidos epigeos usados como termóforos.

Al conjugar las dos líneas de evidencias hemos propuesto que los montículos son la consecuencia de un comportamiento recursivo que involucró el uso del fuego y que se repitió en un mismo lugar a través del tiempo. ¿Qué comportamiento cultural pudo ser el responsable de la acumulación persistente y secular de sedimentos termoalterados? El empleo de $\mathrm{HT}$ es una práctica recurrente que al repetirse en un mismo lugar provoca grandes acumulaciones de residuos termoalterados (Black y Thoms, 2014; Thoms, 1989, 2008, 2009). Los oven mounds de Australia (Brockwell, 2006; Jones, Morrison, Roberts y the River Murray and Mallee Aboriginal Corporation, 2017) nos ilustran cómo la repetición de esta práctica por siglos o milenios, utilizando termóforos sedimentarios, puede originar montículos de tierra de formas y dimensiones similares a los de la cuenca de la laguna Merín (CLM).

\section{¿Cuándo y por qué se adquiere la tecnología de los hornos de tierra?}

Los HT aparecen en un momento muy temprano en América del Sur y del Norte, hace 11.000 años (Rivas y Ocampo, 2005; Thoms, 2017). Para Uruguay se identificaron en el sitio Y 58 de Salto Grande a orillas del río Uruguay, departamento de Salto (Guidón, 1989, p. 446) y en el montículo de Yacaré-Cururú, ubicado en el departamento de Artigas (Consens, 2001, figura 7.2). En ambos casos se le atribuyó una antigüedad de 3.000 años (Figura 1).

Aunque es posible cocinar diferentes tipos de alimentos en $\mathrm{HT}$, su uso se ha vinculado principalmente a la cocción de vegetales (geófitos) que acumulan grandes cantidades de carbohidratos como reservas (Thoms, 2009; Wandsnider, 1997, figura 3). Para muchos cazadores-recolectores de diversos climas y hábitats, los geófitos son recursos vegetales importantes, ya que están disponibles por largos períodos, tienen altas tasas de retorno y alta previsibilidad. Su empleo aparece en debates evolutivos sobre cambios dietarios, expansión a ecosistemas novedosos, prácticas para compartir alimentos y división del trabajo (Greaves y Kramer, 2014).

Los carbohidratos, al ser expuestos a temperaturas de cocción relativamente bajas por períodos prolongados, se transforman en azúcares de cadena corta de mayor biodisponibilidad (Leach, 2007; Thoms, 2009; Wandsnider, 1997). El uso de los HT se intensificó en Norteamérica hacia el 4000 AP, cuando los cazadores-recolectores integraron a su dieta cantidades importantes de geófitos. Para Black y Thoms (2014) y Thoms $(1989,2008,2009)$ su alta oferta, previsibilidad temporo-espacial y los requerimientos organizativos para su colecta y procesamiento hizo que su integración a la dieta se acompañara de cambios tecnológicos, demográficos y sociales. Thoms (2009, p. 579) ha propuesto que la generalización de su uso dio origen a la "revolución pre-agrícola de los carbohidratos", seguramente acompañando condiciones climáticas cambiantes.

\footnotetext{
6 Aunque se han hecho múltiples esfuerzos para identificar las capas que habrían correspondido a eventos constructivos puntuales, cuando se dispone de más de un fechado para capas reconocidas se observa que los que proceden de los niveles más profundos son más antiguos y los más superficiales son más modernos, cubriendo períodos muy dilatados (Bracco, 2006; Bracco et al., 2015; Duarte et al., 2017). Los partidarios del modelo de crecimiento por capas no han ensayado cronologías densas para las capas que reconocen. Una excepción podría ser Milheira y colaboradores $(2019,2018)$, pero ellos no identifican capas.
} 
Los geófitos que se destacan en la CLM son Canna glauca (achira), Typha dominguensis y Typha latifolia (totoras) (Tabla 3). Estos crecen en las formaciones vegetales del Tirirical y del Totoral. Dichas formaciones se desarrollan en suelos permanente o temporalmente anegados y en planicies de inundación de lagunas y arroyos (Barreneche y Zarucki, 2017). Esos ambientes, en cuyo borde se encuentran los montículos (Figura 6), tienen

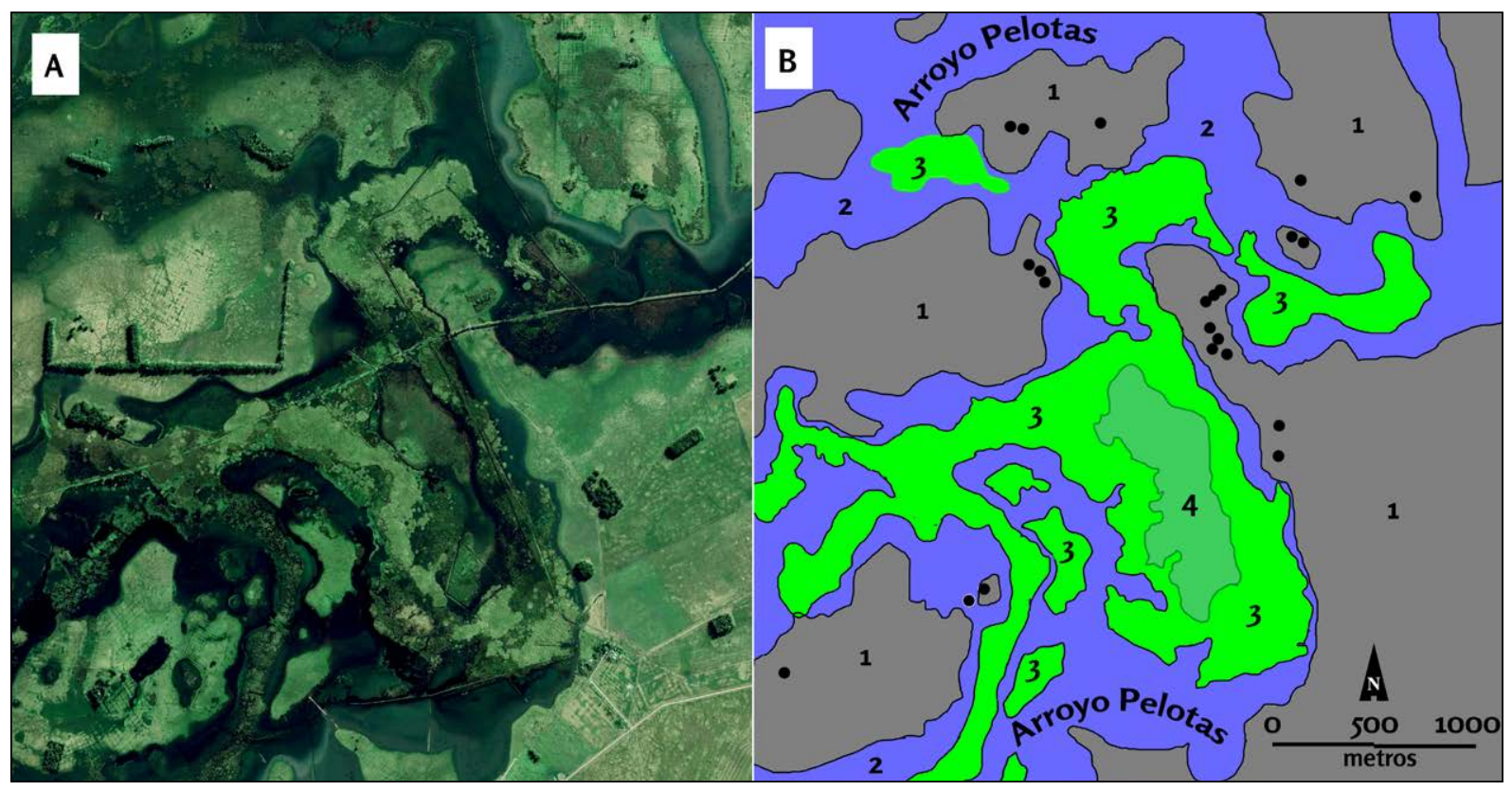

Figura 6. A) Imagen satelital del sitio Pelotas (Google Earth 7/28/2010). B) Esquema de la distribución de la vegetación dominante: 1) Pradera (planicies medias); 2) Plantas acuáticas arraigadas con hojas flotantes (bañado permanente); 3) Tirirical (bañado semipermanente); 4) Totoral (bañado semipermanente). Los puntos negros indican la ubicación de los montículos.

\begin{tabular}{|c|c|c|c|}
\hline Familia & Especie & Parte consumida & Procesamiento según fuentes \\
\hline Apiaceae & Eryngium pandanifolium & Rizoma & ND \\
\hline Arecaceae (1) & Syagrus romanzoffianum & Médula, Fruto & Médula se muele, se cierne y se cocina como pan \\
\hline Arecaceae (1) & Butia odorata & Fruto & ND \\
\hline Bromeliaceae (1) & Bromelia antiacantha & Raíz & ND \\
\hline Cannanaceae (1) & Canna glauca & Rizoma & Asada o hervida \\
\hline Cyperaceae (1) & Cyperus esculentus & Raíz & Asado y hervido \\
\hline \multirow{2}{*}{ Gramineae } & Bambus atrinii & Médula & ND \\
\hline & Echinochloa cruz-galli & Planta & ND \\
\hline \multirow{3}{*}{ Oxalidaceae } & Oxalis brasiliensis & Bulbo & Crudo o asado \\
\hline & Oxalis perdicaria & Bulbo & Crudo o asado \\
\hline & Oxalis sellowiana & Bulbo & Crudo o asado \\
\hline Phytolaccaceae & Phytolacca & Raíz & ND \\
\hline Portulacaceae & Portulaca oleraceae & Planta & Cruda o cocida \\
\hline Typhaceae (1) & Typha domingensis & Rizoma & Hervido, asado al rescoldo \\
\hline Typhaceae (1) & Typha latifolia & Rizoma & Hervido, asado al rescoldo \\
\hline Vitaceae & Cissus palmata & Raíz & Hervida varias veces \\
\hline
\end{tabular}

Tabla 3. Recursos vegetales silvestres presentes en la CLM cuyo consumo está referido en fuentes etnográficas y que por sus características pueden ser cocidos en HT. Referencias: (1) Recursos cuyos silicofitolitos o almidones se han identificado en el registro arqueológico (modificado de del Puerto, 2011b, tablas 1 y 2). 
una amplia extensión regional y la habrían tenido también en el pasado incluso en condiciones de menor precipitación (Bracco et al., 2012). En América el manejo y/o cultivo de Canna glauca está registrado en fuentes etnográficas y datos arqueológicos (del Puerto, 2011b; Piperno, 2011; Ugent, Pozorski y Pozorski, 1984; Watling, Saunaluoma, Pärssinen y Schaan, 2015). Junto con la totora se les reconoce como recursos de gran valor alimenticio (del Puerto, 2011a; Rapoport, Marzocca y Drausal, 2009). El 22\% del peso de la totora - con una productividad que llega a las $7 \mathrm{t} / \mathrm{ha}$ - puede aprovecharse como harina con un valor nutricional similar a la del trigo (Revedin et al., 2010, tabla 2). Silicofitolitos y almidones de ambos géneros se han recuperado en artefactos y en la matriz de los montículos (Tabla 3).

\section{¿Hubo una "revolución de carbohidratos" en la cuenca de la Laguna Merín?}

El sector sur de la CLM carece de un registro arqueológico que permita visualizar con detalle el proceso que desembocó en la elevación de los montículos; sin embargo, algunos indicadores muestran una situación muy distinta durante el Holoceno temprano. El sitio Rincón de los Indios (Figura 1) es el único en el sur de la CLM que ha presentado un registro anterior al Holoceno medio. Sus niveles inferiores, por debajo de los niveles correspondientes a sus dos montículos, han producido dataciones radiocarbónicas en torno a los 8500 años AP y en ellos se ha recuperado un conjunto de puntas de proyectil pedunculadas, desechos de talla bifaciales y artefactos con filo. Las materias primas de estos artefactos proceden de áreas de aprovisionamiento ubicadas entre 8 y $250 \mathrm{~km}$, mostrando un "amplio rango de movilidad y selectividad" (Duarte, 2014, pp. 70-74) o circulación de bienes. Esto contrasta con los conjuntos artefactuales de los montículos, donde se observa la explotación de recursos líticos locales, disponibles en un radio menor a $10 \mathrm{~km}$ (Duarte, 2014). El uso de materias primas locales y tecnología lítica expeditiva ya se había observado para los montículos del sitio CH2D01, bañado de San Miguel (Curbelo y Martínez, 1991, figura 1). El componente inferior de Rincón de los Indios ha sido interpretado como un campamento base de grupos cazadoresrecolectores para los cuales las actividades cinegéticas se habrían enfocado en presas grandes y medianas, diferenciándose de los componentes posteriores donde habría dominado la captura y recolección de recursos locales presentes en el bañado contiguo (López Mazz, Nadal, Suárez Villagrán, De León y Salvo, 2009). Si a ello le sumamos el contraste entre el hallazgo de un solo sitio con un componente pre-montículo y los miles de éstos que se han relevado en la región, debemos reconocer no sólo un cambio en las estrategias de abastecimiento, sino también en la intensidad del uso del espacio. Esto ha sido señalado por Thoms (2009, p. 588) como uno de los indicadores de la "revolución de los carbohidratos".

\section{La teoría de construcción de nicho (TCN)}

Dentro del modelo de la teoría estándar de la evolución, la adaptación se plantea como una relación asimétrica en donde la presión selectiva sobre los organismos es siempre impuesta desde el medio (Lewontin, 2001; Williams, 1996). Una de las limitaciones de este modelo es que desestima cualquier posibilidad de retroalimentación entre los organismos y el ambiente (Odling-Smee, 2009). Sin embargo, los organismos modifican su entorno, imponiendo nuevas presiones selectivas a las nóveles generaciones y a otras especies. Aunque continúa siendo polémico para algunos autores (Mclnerny y Etienne, 2012), el concepto de "nicho ecológico" es central en ecología y en evolución (Chase y Leibold, 2003; Wake, Hadly y Ackerlya, 2009) y ayuda a entender este proceso. Se trata de un concepto de larga data que ha ido modificando su definición a lo largo del último siglo. En 1910, Johnson -a quien se le considera su creador-, lo introdujo 
por primera vez: "se espera que las diferentes especies en una región ocupen diferentes nichos en el ambiente" (Johnson, 1910, p. 87, traducción del autor). Posteriormente Grinnell lo definió como "la menor unidad espacial, en la que una especie puede mantenerse" (Grinnell, 1917, p. 431, traducción del autor). En el año 1927, Elton fue más preciso en la definición del concepto, aportando que el "nicho era el lugar que ocupaba el organismo en la comunidad, su espacio de sobrevivencia, de alimentación, de reproducción y de la relación con sus depredadores" (Elton, 2001, p. 64, traducción del autor). Hutchinson (1957, p. 416) definió nicho como un "hipervolumen" que permite que una especie exista indefinidamente. Posteriormente Maguire Jr. (1973) señaló que el concepto de hipervolumen estaría conformado por diferentes hipervolúmenes demográficos, un hipervolumen reproductivo y un hipervolumen de sobrevivencia. En el presente trabajo utilizaremos el concepto que Laland, Odling-Smee y Feldman (2000, p. 31) sostienen sobre la TCN: "Niche construction refers to the activities, choices, and metabolic processes of organisms, through which they define, choose, modify, and partly create their own niches" ${ }^{\prime \prime}$. Esta definición es coincidente con las anteriormente descritas, agregando que los propios organismos son capaces de modificar y crear su propio nicho. Una de las especies más utilizadas para ejemplificarlo son los castores, que al construir presas transforman indirectamente el patrón y las fuerzas de selección (Odling-Smee, Laland y Feldman, 2003). Estas alteraciones persisten como un legado e inciden en la selección de las generaciones posteriores de castores y de no castores. Esta realidad llevó a acuñar el término de "especies ingenieras" para aquéllas que, como los castores e incluso hormigas, dominan la evolución de los ecosistemas (Jones, Lawton y Shachak, 1994, p. 379). Es evidente que los seres humanos son agentes de cambios ambientales al producir modificaciones en su medio de forma voluntaria o no, las que pasan frecuentemente a formar parte de la estructura del paisaje $y$, en consecuencia, transmiten escenarios y presiones selectivas a las generaciones futuras. La utilización de la TCN en la arqueología es una de las vías para superar las interpretaciones lineales simples que hacen jugar forzantes climáticas o ambientales como únicas causas, ya que incorpora las múltiples e intrincadas transformaciones que produce el accionar humano como impulsoras de la evolución ambiental, biológica y cultural.

Desde este encuadre teórico Laland et al. (2000) proponen un marco evolutivo triple, distinguiendo los sistemas de herencia genética, cultural y ecológica. Los dos primeros han sido ampliamente reconocidos y atendidos, no así el tercero, que remite a los comportamientos influenciados genética o culturalmente y que, al modificar el ambiente en forma perdurable, lo transforman en una herencia ecológica que se perpetúa a escala multigeneracional.

En particular, al desarrollar técnicas de subsistencia -y por tanto elecciones dietarias que van más allá de la estructura natural de la comunidad biótica- los humanos hemos sido siempre agentes activos en la modelación de los ecosistemas (Smith, 2011). La invención o adquisición de las técnicas de subsistencia implican cambios en las presiones de selección evolutiva, aportando nuevas capacidades y beneficios, incluyendo un mayor control y diversidad dietética, que redundan en la posibilidad de obtener mayor cantidad de nutrientes y por ende de energía. Por el otro lado, los sistemas de subsistencia producen frecuentemente modificaciones persistentes en el ambiente. Wollstonecroft (2011) lo señala como la principal causa de construcción de nichos antrópicos diferenciales. Aunque hay múltiples ejemplos de cómo la construcción de nuevos nichos ha producido respuestas adaptativas biológicas (ver Laland et al., 2000), no necesariamente se acompañaron de ellas; no obstante, siempre redundaron en modificaciones socioeconómicas, culturales (Laland y O'Brien, 2010; Laland et

7 La construcción de nicho se refiere a las actividades, elecciones y procesos metabólicos de los organismos, a través de los cuales definen, eligen, modifican y crean en parte sus propios nichos. 
al., 2000) y en transformaciones ambientales persistentes. Las investigaciones de Erickson (2008) y Balée y Erickson (2006) en la Amazonia nos ilustran sobre ello. Si admitimos que los montículos son la consecuencia material del empleo por milenios de una técnica de procesamiento de alimentos, entonces ejemplifican el proceso de la construcción de un nuevo nicho, no sólo basado en relaciones tróficas sino principalmente en una herencia ecológica que retroalimentó el proceso.

\section{¿Cómo crecieron los montículos y se transformó el paisaje?}

Uno de los elementos clave en el funcionamiento de los HT son los termóforos. Luego de haber sido expuesto al fuego disipan calor lentamente asegurando la cocción. Los elementos más utilizados en Europa y América del Norte como termóforos han sido piedras (Black y Thoms, 2014, figura 3; Thoms, 2017). En algunas regiones de Australia donde no hay disponibilidad de rocas, se recurrió a bolas de sedimento o fragmentos de termiteros (Brockwell, 2006, pp. $48-49$ y 52; Campanelli, Muir, Mora, Clarke y Griffin, 2018). Evidencias del uso de termiteros como termóforos fueron halladas para el Holoceno temprano en el sitio de Lapa do Santo, centro este de Brasil (Villagran, Strauss, Alves y Oliveira, 2019). A partir de estos se pueden obtener trozos cementados con buenas propiedades refractarias y bajos niveles de materia orgánica y humedad, lo cual redunda en un menor esfuerzo de confección, buen desempeño térmico, no aporte de humos desagradables y ahorro de combustible (Bracco et al., 2019c). En los montículos de la región de India Muerta/Paso Barranca los fragmentos de tierra quemada -que llegan a constituir el $30 \%$ de la matriz y el restante $70 \%$ es sedimento franco arcillo limoso (Bracco et al., 2019b) - presentan galerías y rasgos microestructurales que indican su procedencia: nidos epigeos construidos por la hormiga Camponotus punctulatus (Bracco et al., 2019c).

La exposición al fuego produce el deterioro de los termóforos. Efectivamente, donde se utilizaron piedras el resultado fue fragmentación (ver Black y Thoms, 2014, figuras 1 a 7). En tanto que donde se emplearon termóforos sedimentarios el resultado fue el descarte de nódulos de tierra quemada o directamente sedimento termoalterado. El volumen de los termóforos desechados está determinando por el tamaño y la cantidad empleados en cada evento de cocción ${ }^{8}$, el número de eventos y la tasa de descarte. Reparando en que cocinar es una actividad cotidiana, debemos admitir que los volúmenes de termóforos descartados a lo largo del tiempo tuvieron que ser importantes. Si la recursividad de procesar alimentos en HT integró un motivo y/o razón para que se repitiese en un mismo lugar, la consecuencia tiene que haber sido grandes acumulaciones de sus desechos. Varios pueden ser los motivos para que un espacio sea reutilizado y se transforme en una estación de HT. En primera instancia, su emplazamiento está vinculado a la ubicación de los recursos que se cocinan (Thoms, 2008). Los vegetales silvestres generalmente se encuentran disponibles en los mismos espacios año tras año, lo que promueve que los lugares de procesamiento tengan poca variabilidad. Por otra parte, los materiales descartados tienen características que las hacen apropiadas para instalar sobre ellos HT. Un sustrato de material refractario, permeable (por lo tanto más seco) y con baja cantidad de materia orgánica, conlleva un ahorro de combustible y no produce humos desagradables (Black y Thoms, 2014). Particularmente en áreas sujetas a inundaciones el amontonamiento (amontonamiento = montículo; ver Ingold, 2013) provoca espacios topográficamente más altos y relativamente más secos, más propicios para habitar, desarrollar actividades de procesamiento de alimentos y también otras. Estos montículos no sólo favorecieron a los humanos, sino que también sus características edáficas y su aislamiento relativo de las inundaciones propician la colonización por parte de plantas y animales, 
frecuentemente ausentes o presentes en menor densidad en la matriz circundante (Figura 7; Bracco et al., 2019b). Actualmente la matriz natural del bañado ha sido remplazada por un barbecho de arroz. La composición y agregación de la vegetación arbórea de los montículos ${ }^{9}$ contrasta marcadamente con la matriz (originalmente bañado).

La instalación de estaciones de HT en múltiples puntos y su permanencia prolongada -conjugando procesos culturales y naturales- se transformó en un rasgo distintivo de amplios sectores de la CLM: una herencia ecológica. Una transmisión de modificaciones ambientales causadas por las generaciones precedentes que no sólo habrían facilitado la producción y reproducción del sistema de subsistencia que las generó, sino que también conllevó modificaciones en la distribución y circulación de la biota lo que coadyuvó en una nueva estructuración del paisaje con refugios y condiciones ambientales que incrementaron la diversidad tanto vegetal como animal, y generó nuevos parches en la matriz de los humedales.

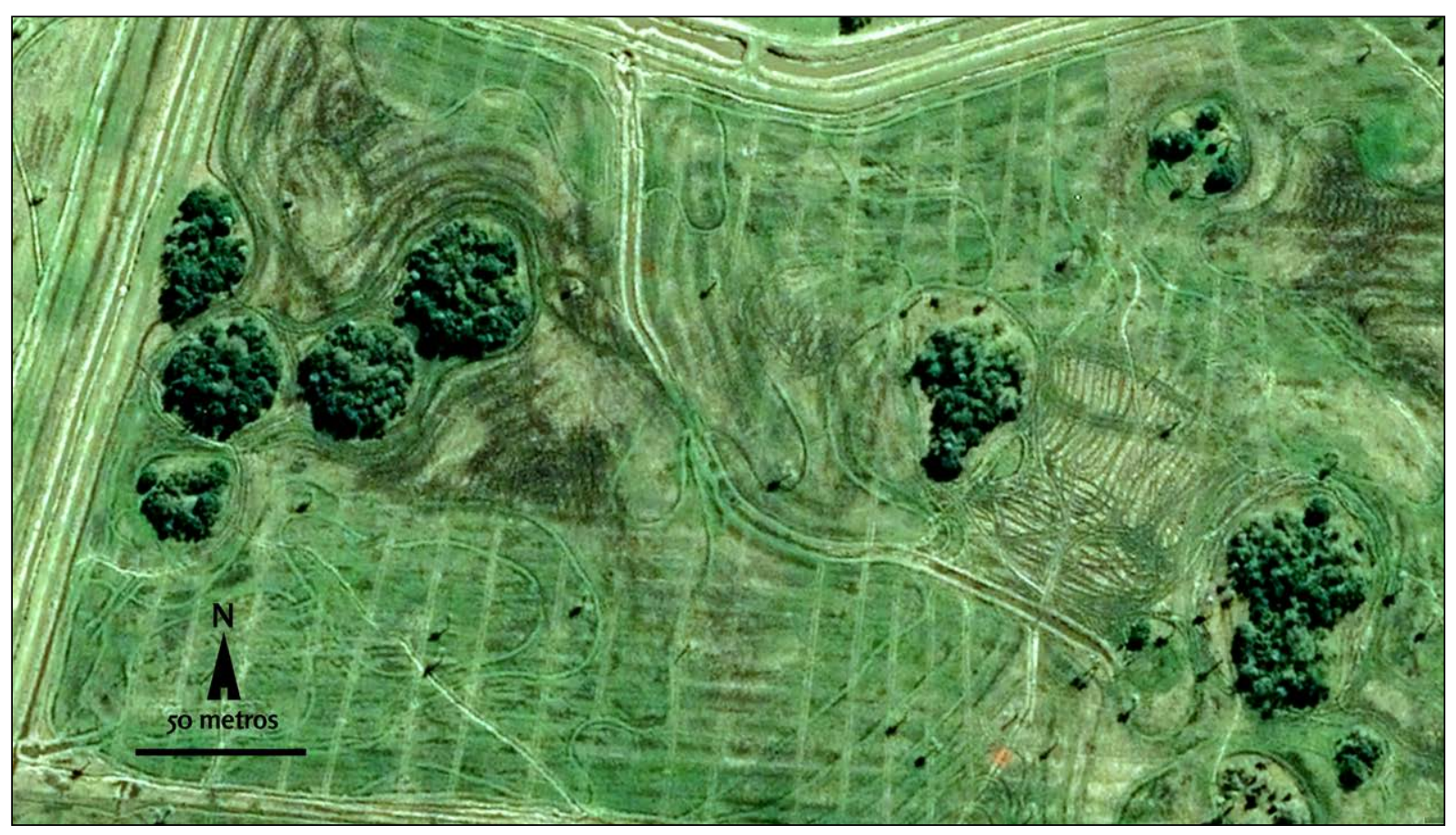

Figura 7. Conjunto de montículos de la región de India Muerta/Paso Barranca (Imagen Google Earth 09/04/2019).

\section{Consideraciones finales}

La función de los montículos, así como los mecanismos que llevaron a su elevación, han sido dos temas centrales de la arqueología de la CLM. La hipótesis que sostiene su crecimiento por la acumulación de desechos producidos por HT replantea ambos temas, al tiempo que habilita o impone abordajes metodológicos nuevos. En consonancia, hemos ensayado en este artículo el empleo del concepto de poscosecha y el andamiaje de la teoría de construcción de nichos. El primero ha permitido proponer cuáles habrían sido los principales recursos procesados en los $\mathrm{HT}$ en función de sus propiedades biológicas: principalmente geófitos. La explotación y consumo de estos

9 Localmente a los montículos cubiertos de vegetación arbórea se les denomina "islas" en referencia a que son islas de monte. 
vegetales -que se pueden cosechar por largos períodos durante el año, tienen altas tasas de retorno y alta previsibilidad - no sólo estarían señalados por el uso de HT, sino también la ubicación de los montículos; una cualidad característica y diferencial de este fenómeno cultural es consistente con su aprovechamiento, así como también lo es su dispersión espacial a lo largo del tiempo. A partir del 2500 AP se produjo un aumento de las precipitaciones (Bracco et al., 2011) y, en forma concomitante, los montículos comienzan a expandirse a zonas más próximas a la laguna Merín acompañando la expansión de los bañados dulceacuícolas y, por ende, de los geófitos de agua dulce. Silicofitolitos y almidones procedentes de artefactos y de la matriz señalan su consumo, al igual que los registros isotópicos dietarios. Su integración a la dieta no sería un fenómeno singular de la CLM; efectivamente, se ha señalado que ca. 4000 años AP su consumo se habría generalizado a escala continental también en América del Norte, junto a una intensificación del uso del espacio y de cambios organizativos.

Por otra parte, la TCN ofrece un marco teórico que permite superar propuestas lineales simples que hacen jugar exclusivamente forzantes climáticas o ambientales como causa del desarrollo de los montículos. La TCN lleva a reconocer como impulsores de la evolución ambiental, biológica y cultural las múltiples e intrincadas transformaciones que produce el accionar humano en el ambiente. Si los montículos se formaron a partir de la adopción de un nuevo sistema de subsistencia, el que incluyó necesariamente el establecimiento de nuevas relaciones tróficas, son un ejemplo de la construcción de un nicho humano diferencial. Éste - por las opciones tecnológicas involucradasintegró la construcción de una herencia ecológica -modificaciones permanentes en el entorno y dentro de ellas particularmente los montículos- que incidió en la producción y especialmente en la reproducción del sistema. Esta transformación del ambiente también habría sido un disparador para que algunas especies -animales y vegetales - pudieran adaptarse exitosamente a las nuevas condiciones (e.g. coronillas y talas observados generalmente sobre los cerritos), lo que ejemplifica cómo la presión selectiva frecuentemente está determinada por procesos de retroalimentación entre los organismos y el ambiente (Lewontin, 2001; Williams, 1996), donde la presión selectiva sobre los organismos es siempre impuesta desde el medio tanto intrínseco (e.g. nuevas prácticas culturales, comportamiento de especies, cambio de uso del suelo) como externo (e.g. clima). Futuros estudios aportarán datos más relevantes al respecto.

Los montículos no son una realidad arqueológica exclusiva de la CLM: los hallamos en regiones próximas, delta del Paraná-bajo Río Uruguay (Argentina-Uruguay) en el Pantanal (Brasil) y en otras partes de América del Sur. Seguramente no tuvieron la misma génesis. Nuevas hipótesis sobre sus procesos de elevación y visiones alternativas sobre su funcionamiento en el paisaje, pueden mejorar nuestro entendimiento sobre la necesaria diversidad de un fenómeno cultural que caracteriza amplias áreas de las tierras bajas sudamericanas y un amplio período de su prehistoria.

\section{Agradecimientos}

Agradecemos al Laboratorio de Radioquímica del Centro de Investigaciones Nucleares (CIN), Facultad de Ciencias, Universidad de la República, Uruguay, por su colaboración. También queremos expresar nuestro reconocimiento a los evaluadores anónimos que con sus comentarios y sugerencias permitieron mejorar la calidad del trabajo. 


\section{Referencias citadas}

» Ambrose, S. H. (1990). Preparation and characterization of bone and tooth collagen for isotopic analysis. Journal of Archaeological Science, 17(4), 431-451. https://doi.org/10.1016/03054403(90)90007-R

» Arechavaleta, J. (1892). Viaje a San Luis. En El Uruguay en la exposición histórico-americana de Madrid. Memoria de los trabajos realizados por la comisión Nacional encargada de organizar los elementos de concurrencia (pp. 65-106). Montevideo: Imprenta Artística de Dornaleche y Reyes.

» Baeza, J. y Panario, D. (1999). La horticultura indígena en las estructuras monticulares. En Actas de las Primeras Jornadas sobre Cenozoico en Uruguay. Montevideo: SUG, INGEPA y UNCIEP, Facultad de Ciencias.

» Balée, W. L. y Erickson, C. L. (Eds.). (2006). Time and Complexity in Historical Ecology: Studies in the Neotropical Lowlands. Nueva York: Columbia University Press.

» Barreneche, J. M. y Zarucki, M. (2017). Mapeo y clasificación de formaciones vegetales del sitio Ramsar Bañados del Este y Franja Costera. Rocha: Programa de Conservación de la Biodiversidad y Desarrollo Sustentable en los Humedales del Este (PROBIDES).

» Black, S. L. y Thoms, A. V. (2014). Hunter-Gatherer earth ovens in the archaeological record: fundamental concepts. American Antiquity, 79(2), 204-226. https://doi. org/10.7183/0002-7316.79.2.204

" Bracco, R. (2006). Montículos de la cuenca de la laguna Merín: Tiempo, espacio y sociedad. Latin American Antiquity, 17(4), 511-540. https://doi.org/10.2307/25063070

» Bracco, R., Cabrera, L. y López Mazz, J. M. (2000). La prehistoria de las tierras bajas de la cuenca de la laguna Merín. En A. Duran y R. Bracco Boksar (Eds.), Arqueología de las Tierras Bajas (pp. 13-38). Montevideo: Ministerio de Educación y Cultura, Comisión Nacional de Arqueología.

» Bracco, R., del Puerto, L. e Inda, H. (2008). Prehistoria y arqueología de la cuenca de Laguna Merín. En D. Loponte y A. Acosta (Eds.), Entre la Tierra y el Agua. Arqueología de Humedales de Sudamérica (pp. 1-59). Buenos Aires: AINA.

» Bracco, R., del Puerto, L., Inda, H., Capdepont, I., Panario, D. y García-Rodríguez, F. (2012). Evolución ambiental y constructores de cerritos en la región de India Muerta. Un replanteo. Trabajo presentado en III Jornadas del Cenozoico (pp. 14-15). [CD ROM]. Montevideo.

» Bracco, R., del Puerto, L., Inda, H., Panario, D., Castiñeira, C. y García-Rodríguez, F. (2011). The relationship between emergence of mound builders in SE Uruguay and climate change inferred from opal phytolith records. Quaternary International, 245, 62-73. https://doi.org/10.1016/j. quaint.2010.05.025

» Bracco, R., Duarte, C., Gutiérrez, O., Tassano, M., Bazzino, A. y Panario, D. (2019a). El fuego en la génesis de los montículos de la cuenca de la Laguna Merín. Su visualización a través de las técnicas de datación por luminiscencia. En M. Bonnin, A. Laguens y M. B. Marconetto (Eds.), Libro de Resúmenes del XX Congreso Nacional de Arqueología Argentina - 50 años de Arqueologías (pp. 1536-1541). Córdoba: Universidad Nacional de Córdoba.

» Bracco, R., Duarte, C., Gutiérrez, O., Tassano, M., Norbis, W. y Panario, D. (2020). El fuego en los procesos constructivos de los montículos del sur de la cuenca de la Laguna Merín. Un aporte de la datación por luminiscencia (OSL-TL). Latin American Antiquity, 31(3), 1-19. https://doi. org/10.1017/laq.2019.98

» Bracco, R., Inda, H. y del Puerto, L. (2015). Complejidad en montículos de la cuenca de la laguna Merín y análisis de redes sociales. Intersecciones en Antropología, 16(1), 271-286.

» Bracco, R., Panario, D., Gutiérrez, O., Bazzino, A., Duarte, C., Odino, R. y Reina, E. (2019b). Mounds and landscape in the Merín Lagoon basin, Uruguay. En H. Inda y F. García-Rodríguez (Eds.), Advances in Coastal Geoarchaeology in Latin America (pp. 103-129). Cham: Springer. https://doi.org/10.1007/978-3-030-17828-4_7 
» Bracco, R., Panario, D., Gutiérrez, O., Duarte, C. y Bazzino, A. (2019c). Estructuras monticulares y hormigueros en el sur de la Cuenca de la Laguna Merín: ¿ingenieros ambientales y/o la estrategia del bricoleur? Revista de Antropología del Museo de Entre Ríos, 5(1), 24-40. https:// ppct.caicyt.gov.ar/index.php/antropmuser/article/view/13976

» Bracco, R. y Ures, C. (1999). Ritmos y dinámica constructiva de las estructuras monticulares. Sector sur de la cuenca de la laguna Merín. Uruguay. En J. M. López y M. Sanz (Eds.), Arqueología y Bioantropología de las Tierras Bajas (pp. 13-33). Montevideo: Facultad de Humanidades y Ciencias de la Educación, Universidad de la República.

» Brockwell, S. (2006). Earth mounds In Northern Australia: A review. Australian Archaeology, 63(1), 47-56. https://doi.org/10.1080/03122417.2006.11681837

» Cabrera, L. (2013). Construcciones en tierra y estructura social en el Sur del Brasil y Este de Uruguay (Ca. 4.000 a 300 A.P.). Techne, 1(1), 25-33.

» Cabrera, L. y Marozzi, O. (2001). Las áreas domésticas de los constructores de cerritos: el sitio CG14E01. En Arqueología Uruguaya Hacia el Fin del Milenio. Anales del IX Congreso Nacional de Arqueología (pp. 55-68). Montevideo: Ministerio de Educación y Cultura.

» Campanelli, M., Muir, J., Mora, A., Clarke, D. y Griffin, D. (2018). Re-Creating an aboriginal earth oven with clayey heating elements: experimental archaeology and paleodietary implications. EXARC Journal, 2, 1-14. https://exarc.net/issue-2018-2/ea/re-creating-aboriginal-earthoven-clayey-heating-elements-experimental-archaeology-and-paleodietary (Acceso: 15 de noviembre, 2019).

» Chase, J. M. y Leibold, M. A. (2003). Ecological niches. Linking classical and contemporary approaches. Chicago: University of Chicago Press.

»Consens, M. (2001). Yacaré-Cururú: 18 años después. En Arqueología Uruguaya Hacia el Fin del Milenio. Anales del IX Congreso Nacional de Arqueología (pp. 115-123). Montevideo: Ministerio de Educación y Cultura.

» Copé, S. M. (1991). A ocupação pré-Colonial do Sul e Sudeste do Rio Grande do Sul. En A. L. Jacobus y A. A. Kern (Eds.), Arqueologia pré-histórica do Rio Grande do Sul (pp. 191-219). Porto Alegre: Mercado Aberto.

» Curbelo, C. y Martínez, E. (1991). Aprovechamiento de materias primas líticas para un área relacionada con la sierra de San Miguel, Depto. de Rocha, R.O.U. Estudos Ibero-Americanos, 17(2), 47. https://doi.org/10.15448/1980-864X.1991.2

» del Puerto, L. (2011a). Ponderación de recursos vegetales silvestres del este del Uruguay: rescatando el conocimiento indígena tradicional. Trama. Revista de Cultura y Patrimonio, 3, 22-41.

» del Puerto, L. (2011b). Silicofitolitos como indicadores paleoambientales. Bases comparativas y reconstrucción paleoclimática a partir del Pleistoceno tardío en el SE del Uruguay. Madrid: Editorial Académica Española.

» del Puerto, L. (2015). Interrelaciones humano-ambientales durante el Holoceno tardío en el este del Uruguay: Cambio Climático y Dinámica Cultural. (Tesis Doctoral inédita), PEDECIBA, Universidad de la República, Uruguay.

» del Puerto, L. e Inda, H. (2005). Paleoetnobotánica de los constructores de cerritos del noreste de Uruguay: análisis de silicofitolitos de la estructura monticular YALE27 y su entorno. En C. Gianotti (Comp.), Proyecto de Cooperación Científica: Desarrollo metodológico y aplicación de nuevas tecnologías para la gestión integral del Patrimonio Arqueológico en Uruguay. Traballos de Arqueoloxía e Patrimonio (TAPA), 36, 109-122.

» DeNiro, M. J. (1985). Postmortem preservation and alteration of in vivo bone collagen isotope ratios in relation to paleodietary reconstruction. Nature, 317, 806-809. https://doi. org/10.1038/317806a0

» Duarte, C. (2014). Una aproximación a las áreas de aprovisionamiento de los grupos prehistóricos del sitio "Rincón de los Indios» (Dpto. Rocha). (Tesis de Licenciatura inédita), Universidad de la República: Uruguay. 
» Duarte, C., Bracco, R., Panario, D., Tassano, M., Cabrera, M., Bazzino, A. y del Puerto, L. (2017). Datación de estructuras monticulares por OSL/TL. Revista de Antropología del Museo de Entre Ríos, 3(1), 14-26. https://ppct.caicyt.gov.ar/index.php/antropmuser/article/view/12057

»Elton, C. S. (2001). Animal Ecology. Chicago: The University of Chicago Press.

» Erickson, C. L. (2008). Amazonia: The historical ecology of a domesticated landscape. En H. Silverman y W. Isbell (Eds.), The Handbook of South American Archaeology (pp. 157-183). New York, NY: Springer. https://doi.org/10.1007/978-0-387-74907-5_11

»Ferrés, C. (1927). Los «terremotos de los indios». Revista de la Sociedad Amigos de la Arqueología, 1, 139-149.

» Francey, R. J., Manning, M. R., Allison, C. E., Coram, S. A., Etheridge, D. M., Langenfelds, R. L., Lowe, D. C. y Steele, L. P. (1999). A history of $\delta 13 \mathrm{C}$ in atmospheric $\mathrm{CH} 4$ from the Cape Grim Air Archive and Antarctic firn air. Journal of Geophysical Research: Atmospheres, 104(D19), 23631-23643. https://doi.org/10.1029/1999JD900357

》 Gianotti, C. (2000). Paisajes monumentales en la región meridional sudamericana. Gallaecia, $19,43-72$.

» Gianotti, C. y Bonomo, M. (2013). De montículos a paisajes: procesos de transformación y construcción de paisajes en el sur de la cuenca del Plata. Comechingonia Virtual: Revista Electrónica de Arqueología, 17(2), 129-163. http://hdl.handle.net/11336/ 13622\%0Ahttp://ref.scielo.org/yc82vx (Acceso: 20 de julio, 2019)

" Gianotti, C., Criado Boado, F., Seoana, Y., Cancela, C., Piñeiro, G., Gazzán, N. y Capdepont, I., (2009). Dinámica constructiva y formación de un asentamiento monumental en el valle de Caraguatá (Tacuarembó). Informes y Trabajos, 3, 265-274.

» Gianotti, C., del Puerto, L., Inda, H. y Capdepont, I. (2013). Construir para producir. Pequeñas elevaciones en tierra para el cultivo de maíz en el sitio Cañada de los Caponcitos, Tacuarembó (Uruguay). Cuadernos del Instituto Nacional de Antropología y Pensamiento Latinoamericano - Series Especiales, 1(1), 12-25.

» Giddens, A. (1997). Modernidad e identidad del yo: el yo y la sociedad en la época contemporánea. Madrid: Península.

» Greaves, R. D. y Kramer, K. L. (2014). Hunter-gatherer use of wild plants and domesticates: archaeological implications for mixed economies before agricultural intensification. Journal of Archaeological Science, 41, 263-271. https://doi.org/10.1016/j.jas.2013.08.014

» Grinnell, J. (1917). The niche-relationships of the California Thrasher. The Auk, 34(4), 427-433. https://doi.org/10.2307/4072271

» Guidón, N. (1989). Misión de Rescate Arqueológico de Salto Grande, República Oriental del Uruguay (Tomo II, 1era. Parte). Montevideo: Ministerio de Educación y Cultura

» Hedges, R. E. M. y Reynard, L. M. (2007). Nitrogen isotopes and the trophic level of humans in archaeology. Journal of Archaeological Science, 34(8), 1240-1251. https://doi.org/10.1016/j. jas.2006.10.015

» Hutchinson, G. E. (1957). Concluding remarks. Cold Spring Harbor Symposia on Quantitative Biology, 22, 415-427.

» Inglett, P. W. y Reddy, K. R. (2006). Investigating the use of macrophyte stable C and N isotopic ratios as indicators of wetland eutrophication: Patterns in the P-affected Everglades. Limnology and Oceanography, 51(5), 2380-2387. https://doi.org/10.4319/lo.2006.51.5.2380

» Ingold, T. (2013). Making Anthropology, Archaeology, Art and Architecture. Londres: Routledge. https://doi.org/10.4324/9780203559055

» Iriarte, J. (2006). Landscape transformation, mounded villages and adopted cultigens: the rise of early Formative communities in south-eastern Uruguay. World Archaeology, 38(4), 644663. https://doi.org/10.1080/00438240600963262

» Iriarte, J. (2007). La construcción social y transformación de las comunidades del Periodo Formativo Temprano del sureste de Uruguay. Boletín de Arqueología PUCP, 11, 143-166. 
» Iriarte, J., Holst, I., López Mazz, J. M. y Cabrera, L. (2000). Subtropical wetland adaptation in Uruguay during the mid-Holocene: An archaeobotanical perspective. En B. A. Purdy (Ed.), Enduring Records: the Environmental and Cultural Heritage of Wetlands (pp. 61-70). Oxford: Oxbow Books.

» Iriarte, J., Holst, I., Marozzi, O., Listopad, C., Alonso, E., Rinderknecht, A. y Montaña, J. R. (2004). Evidence for cultivar adoption and emerging complexity during the mid-Holocene in the La Plata basin. Nature, 432(7017), 614-617. https://doi.org/10.1038/nature02983

» Johnson, R. H. (1910). Determinate evolution in the color-pattern of the lady-beetles. Washington, D.C.: Carnegie Institution of Washington. https://doi.org/10.5962/bhl.title.30902

» Jones, C. G., Lawton, J. H. y Shachak, M. (1994). Organisms as ecosystem engineers. Oikos, 69(3), 373-386. https://doi.org/10.1007/978-1-4612-4018-1_14

» Jones, R., Morrison, M., Roberts, A. y the River Murray and Mallee Aboriginal Corporation. (2017). An analysis of Indigenous earth mounds on the Calperum Floodplain, Riverland, South Australia. Journal of the Anthropological Society of South Australia, 41, 18-61.

» Laland, K. N. y O'Brien, M. J. (2010). Niche construction theory and archaeology. Journal of Archaeological Method and Theory, 17(4), 303-322. https://doi.org/10.1007/s10816-0109096-6

» Laland, K. N., Odling-Smee, F. J. y Feldman, M. W. (2000). Niche construction, biological evolution, and cultural change. Behavioral and Brain Sciences, 23, 131-175. https://doi. org/10.1017/S0140525X00002417

" Leach, J. D. (2007). Prebiotics in ancient diets. Food Science and Technology Bulletin: Functional Foods, 4(1), 1-8.

» Lewontin, R. C. (2001). The Triple Helix: Gene, Organism, and Environment. Cambridge: Harvard University Press.

» López Mazz, J. M. (2000). Trabajos en tierra y complejidad cultural en las Tierras Bajas del Rincón de los Indios. En A. Duran y R. Bracco (Eds.), Arqueología de las Tierras Bajas (pp. 271-285). Montevideo: Ministerio de Educación y Cultura, Comisión Nacional de Arqueología.

» López Mazz, J. M. (2001). Las estructuras tumulares (cerritos) del litoral atlántico uruguayo. Latin American Antiquity, 12(3), 231-255. https://doi.org/10.2307/971631

» López Mazz, J. M. y Bracco, R. (1992). Relación Hombre-Medio Ambiente en las poblaciones prehistóricas de la Zona Este del Uruguay. En O. Ortiz-Troncoso y T. van der Hammen (Eds.), Archaeology and Environment in Latin America (pp. 259-282). Amsterdam: Instituut voor Preen Protohistorische Archeologie Albert Egges van Giffen, Universiteit van Amsterdam.

» López Mazz, J. M., Nadal, O., Suárez Villagrán, X., De León, V. y Salvo, X. (2009). La gestión regional de los recursos minerales en las tierras bajas del este: el espacio como variable de la producción lítica. En A. Gascue y J. M. López Mazz (Eds.), La arqueología prehistórica uruguaya en el siglo XXI (pp. 85-100). Montevideo: Biblioteca Nacional - Facultad de Humanidades y Ciencias de la Educación.

» López Mazz, J. M., Rostain, S. y McKey, D. (2016). Cerritos, tolas, tesos, camellones y otros montículos de las Tierras Bajas de Sudamérica. Revista de Arqueología, 29(1), 86. https://doi. org/10.24885/sab.v29i1.444

» Loureiro, A. G. (2008). Sítio PT-02-Sotéia: análise dos processos formativos de um cerrito na região sudoeste da Laguna dos Patos/RS. (Tesis de Maestría inédita), Universidade de São Paulo, Brasil. 10.11606/D.71.2008.tde-29042008-151446

» Maguire Jr., B. (1973). Niche response structure and the analytical potentials of its relationship to the habitat. The American Naturalist, 107(954), 213-246. https://doi. org/10.1086/282827

» Mclnerny, G. J. y Etienne, R. S. (2012). Ditch the niche - is the niche a useful concept in ecology or species distribution modelling? Journal of Biogeography, 39(12), 2096-2102. https://doi. org/10.1111/jbi. 12033 
» Milheira, R. G., Attorre, T. y Borges, C. (2019). Construtores de cerritos na Laguna Dos Patos, Pontal da Barra, sul do Brasil: Lugar persistente, território e ambiente construído no Holoceno recente. Latin American Antiquity, 30(1), 35-54. https://doi.org/10.1017/laq.2018.66

» Milheira, R. G. y Gianotti, C. (2018). The Earthen Mounds (Cerritos) of Southern Brazil and Uruguay. En C. Smith (Ed.), Encyclopedia of Global Archaeology (pp. 1-9). Cham: Springer. https://doi.org/10.1007/978-3-319-51726-1_3025-1

» Moreno, F. (2014). La gestión de los recursos animales en la prehistoria del Este de Uruguay (4000 años AP-Siglo XVI). (Tesis Doctoral inédita), Universitat Autònoma de Barcelona, España.

» Mut, P. (2015). Paleodieta de los pobladores prehistóricos del este del Uruguay: un retrato isotópico. Anuario de Arqueología, 2015, 147-178.

» O'Connell, T. C., Kneale, C. J., Tasevska, N. y Kuhnle, G. G. C. (2012). The diet-body offset in human nitrogen isotopic values: a controlled dietary study. American Journal of Physical Anthropology, 149(3), 426-434. https://doi.org/10.1002/ajpa.22140

»Odling-Smee, J. (2009). Niche Construction in Evolution, Ecosystems and Developmental Biology. En A. Barberouss, M. Morange y T. Pradeu (Eds.), Mapping the Future of Biology. Evolving Concepts and Theories (pp. 69-92). Dordrecht: Springer. https://doi. org/10.1007/978-1-4020-9636-5_6

» Odling-Smee, J., Laland, K. N. y Feldman, M. W. (2003). Niche Construction: The Neglected Process in Evolution. Princeton: Princeton University Press.

» Olivero, J. y Campos, S. (2001). Análisis de partículas biosilíceas en la matriz del sitio arqueológico $\mathrm{CH} 2 \mathrm{D01}$, San Miguel, Rocha-Uruguay. En Arqueología Uruguaya Hacia el Fin del Milenio. Anales del IX Congreso Nacional de Arqueología (pp. 539-550). Montevideo: Ministerio de Educación y Cultura.

»Pastor Quiles, M., Knoll, F. y Jover Maestre, F. J. (2019). ¿Adobes, terrones o bolas de barro amasado? Aportaciones para el reconocimiento arqueológico de las distintas técnicas constructivas que emplean módulos de tierra. Arqueología, 25(2), 213-234. https://doi. org/10.34096/arqueologia.t25.n2.6868

»Pintos Blanco, S. (2000). Economía "húmeda" del este del Uruguay: el manejo de los recursos faunísticos. En A. Duran y R. Bracco Boksar (Eds.), Arqueología de las Tierras Bajas (pp. 249270). Montevideo: Comisión Nacional de Arqueología, Ministerio de Educación y Cultura.

»Pintos Blanco, S. y Capdepont, I. (2001). Arqueología en la Cuenca de la Laguna de Castillos. Apuntes sobre complejidad cultural en sociedades cazadoras-recolectoras del Este del Uruguay. ArqueoWeb - Revista sobre Arqueología en Internet, 3(2), 1-15. https://webs.ucm.es/ info/arqueoweb/pdf/3-2/pintos.pdf (Acceso: 20 de setiembre, 2019)

»Piperno, D. R. (2011). The origins of plant cultivation and domestication in the New World Tropics. Current Anthropology, 52(S4), S453-S470. https://doi.org/10.1086/659998

» Rapoport, E. H., Marzocca, A. y Drausal, B. S. (2009). Malezas comestibles del Cono Sur y otras partes del planeta. San Carlos de Bariloche: Instituto Nacional de Tecnología Agropecuaria.

» Revedin, A., Aranguren, B., Becattini, R., Longo, L., Marconi, E., Lippi, M. M., Skakun, N., Sinitsyn, A., Spiridonova, E. y Svoboda, J. (2010). Thirty thousand-year-old evidence of plant food processing. Proceedings of the National Academy of Sciences, 107(44), 18815-18819. https:// doi.org/10.1073/pnas.1006993107

»Rivas, P. y Ocampo, C. (2005). El antiguo curanto chilote. En G. Badal, F. Balcella, M. Montanari, C. Santa Cruz y C. Altamirano (Eds.), Chile, País Oceánico (pp. 70-71). Santiago: Editorial Ocho Libros.

»Schmitz, P. I. (1976). Sítios de Pesca Lacustre em Rio Grande, RS, Brasil. (Tesis de «Livredocente»), Universidade Católica do Rio Grande do Sul,Brasil.

»Schmitz, P. I. (1981). Contribuciones a la prehistoria de Brasil. Pesquisas, Série Antropologia, $32,1-243$. 
» Schmitz, P. I., Girelli, M. y Rosa, A. O. (1997). Pesquisas arqueológicas em Santa Vitória do Palmar, RS. En P. Schmitz (Ed.), Arqueologia do Rio Grande do Sul, Brasil: Documento No 7 (pp. 5-95). São Leopoldo: UNISINOS.

» Schorr, M. H. E. (1975). Abastecimento indígena na área alagadiça lacustre de Rio Grande, Rio Grande do Sul, Brasil. Cadernos de Associação Santanense Pró-Ensino Superior, 1, 1-116.

»Serrentino, C. M. (2013). Cuenca Binacional de la Laguna Merin. México: Centro del agua para América Latina y el Caribe.

» Smith, B. D. (2011). General patterns of niche construction and the management of «wild» plant and animal resources by small-scale pre-industrial societies. Philosophical Transactions of the Royal Society B: Biological Sciences, 366(1566), 836-848. https://doi.org/10.1098/ rstb.2010.0253

"Thoms, A. V. (1989). The northern roots of hunter-gatherer intensification: Camas and the Pacific Northwest (Tesis Doctoral inédita), Washington State University, EE.UU.

" Thoms, A. V. (2008). Ancient savannah roots of the carbohydrate revolution in SouthCentral North America. Plains Anthropologist, 53(205), 121-136. https://doi.org/10.1179/ pan.2008.008

» Thoms, A. V. (2009). Rocks of ages: propagation of hot-rock cookery in western North America. Journal of Archaeological Science, 36(3), 573-591. https://doi.org/10.1016/j.jas.2008.11.016

» Thoms, A. V. (2017). Burned-Rock Features. En A. S. Gilbert (Ed.), Encyclopedia of Earth Sciences Series (pp. 89-94). Dordrecht: Springer. https://doi.org/10.1007/978-1-4020-4409-0

» Ugent, D., Pozorski, S. y Pozorski, T. (1984). New evidence for ancient cultivation of Canna edulis in Peru. Economic Botany, 38(4), 417-432. https://doi.org/10.1007/BF02859081

»Villagran, X. S. y Gianotti, C. (2013). Earthen mound formation in the Uruguayan lowlands (South America): micromorphological analyses of the Pago Lindo archaeological complex. Journal of Archaeological Science, 40(2), 1093-1107. https://doi.org/10.1016/j.jas.2012.10.006

» Villagran, X. S., Strauss, A., Alves, M. y Oliveira, R. E. (2019). Virtual micromorphology: The application of micro-CT scanning for the identification of termite mounds in archaeological sediments. Journal of Archaeological Science: Reports, 24, 785-795. https://doi.org/10.1016/ j.jasrep.2019.02.035

»Wake, D. B., Hadly, E. A. y Ackerlya, D. D. (2009). Biogeography, changing climates, and niche evolution. Proceedings of the National Academy of Sciences of the United States of America, 106(2), 19631-19636. https://doi.org/10.1073/pnas.0911097106

» Wandsnider, L. (1997). The roasted and the boiled: food composition and heat treatment with special emphasis on pit-hearth cooking. Journal of Anthropological Archaeology, 16(1), 1-48. https://doi.org/10.1006/jaar.1997.0303

» Watling, J., Saunaluoma, S., Pärssinen, M. y Schaan, D. (2015). Subsistence practices among earthwork builders: phytolith evidence from archaeological sites in the southwest Amazonian interfluves. Journal of Archaeological Science: Reports, 4, 541-551. https://doi.org/10.1016/j. jasrep.2015.10.014

» Williams, G. C. (1996). Adaptation and Natural Selection: A Critique of Some Current Evolutionary Thought. Princeton: Princeton University Press.

»Wollstonecroft, M. M. (2011). Investigating the role of food processing in human evolution: a niche construction approach. Archaeological and Anthropological Sciences, 3(1), 141-150. https://doi.org/10.1007/s12520-011-0062-3

»Wrangham, R. y Conklin-Brittain, N. (2003). 'Cooking as a biological trait'. Comparative Biochemistry and Physiology. Part A: Molecular \& Integrative Physiology, 136(1), 35-46. https:// doi.org/10.1016/S1095-6433(03)00020-5 